\title{
Yod
}

Revue des études hébraïques et juives

$14 \mid 2009$

La littérature israélienne, miroir d'une société multiple

\section{Les études juives en Roumanie}

\section{Ana Barbulescu et Cristina Ciucu}

\section{(2) OpenEdition}

Journals

Édition électronique

URL : https://journals.openedition.org/yod/124

DOI : 10.4000/yod. 124

ISSN : 2261-0200

Éditeur

INALCO

Édition imprimée

Date de publication : 1 octobre 2009

Pagination : 341-357

ISBN : 978-2-85831-178-1

ISSN : 0338-9316

Référence électronique

Ana Barbulescu et Cristina Ciucu, «Les études juives en Roumanie », Yod [En ligne], 14 | 2009, mis en ligne le 30 octobre 2011, consulté le 08 juillet 2021. URL : http://journals.openedition.org/yod/124 ; DOI : https://doi.org/10.4000/yod. 124

Ce document a été généré automatiquement le 8 juillet 2021.

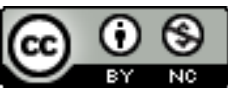

Yod est mis à disposition selon les termes de la Licence Creative Commons Attribution - Pas d'Utilisation Commerciale 4.0 International. 


\title{
Les études juives en Roumanie
}

\author{
Ana Barbulescu et Cristina Ciucu
}

1 La conscience identitaire juive se définit socialement et politiquement en Roumanie entre la deuxième moitié du dix-neuvième siècle et la première moitié du vingtième siècle. La lutte pour les droits sociaux et politiques dans les milieux laïques coïncide avec une démarche de réaffirmation de l'identité culturelle. La nécessité d'une telle démarche assumée est en partie - en Roumanie comme ailleurs - le résultat des conditions difficiles et de la prise de conscience d'une intégration illusoire. Elle devient encore plus pesante après 1922, avec la violation répétée du traité international des minorités ${ }^{1}$ et l'instauration d'une politique antisémite officielle.

2 La communauté juive de Roumanie constitue lors de l'unification roumaine, en 1918², approximativement $30 \%$ de la population roumaine allogène. En 1920 elle représente $5,3 \%$ de la population totale et selon le recensement de 1930 seulement $4 \%$. Il s'agit d'une population en majorité urbaine $-13,6 \%$ de la population des villes et seulement $1,4 \%$ de la population des villages ${ }^{3}$ - qui manifeste un important degré d'assimilation linguistique (28,8\% en 1930). Les Juifs de Transylvanie ne parlaient en grande partie que le hongrois et avaient d'ailleurs été comptabilisés par les recensements hongrois qui ne comportaient, de manière délibérée, que des critères linguistiques et religieux comme hongrois ${ }^{4}$.

La nécessité d'accéder à l'éducation laïque se manifeste visiblement dans les provinces historiques roumaines déjà dans la première moitié du dix-neuvième siècle. Les Juifs sont officiellement admis dans les écoles publiques à partir de 1831. Mais des lois qui limitent l'accès des allogènes ne tardent pas à être émises - par exemple celle de 1893 limitant l'accès à l'enseignement public primaire pour les populations minoritaires. Cette loi est étendue en 1898 à l'enseignement secondaire et supérieur. Comme une alternative, des écoles juives laïques commencent à fonctionner à partir de 1851 à Bucarest et de 1852 à Iasi. Il s'agit d'institutions financées par la communauté qui comprenaient outre un enseignement général, un enseignement de culture juive et de langue hébraïque ${ }^{5}$. L'accès à l'enseignement public n'étant pas toujours facile, on assiste à l'apparition des "écoles israélites-roumaines » où l'instruction primaire et secondaire étaient en roumain, mais où on enseignait aussi l'hébreu et le Talmud Tora. 
Même si ces écoles n'avaient pas le droit d'accorder des diplômes officiels, elles ont eu une contribution majeure dans la transmission et la dissémination de la culture juive dans les milieux juifs non religieux, tout en contribuant à maintenir une cohésion intracommunautaire.

Les écoles publiques juives connaissent des crises (entre 1870 et 1893) à cause des problèmes financiers des communautés et de la concurrence - avant les mesures restrictives des autorités roumaines - des écoles publiques ${ }^{6}$. Mais avec la restriction de l'accès des enfants juifs aux écoles publiques, le réseau d'écoles juives s'épanouit et se diversifie (écoles secondaires, de métiers, de filles, etc.).

Dans la période d'entre les deux guerres commencent à fonctionner des réseaux d'institutions juives d'enseignement en hébreu, appelées Tarbut, comprenant des écoles maternelles, écoles primaires, secondaires et lycées. Entre 1923 et 1935 fonctionnait à Chisinau un lycée hébraïque orthodoxe, appelé Magen David. Le fonctionnement de toutes ces institutions cesse ou devient très précaire à partir de l'année 1940.

6 Dans ce contexte, la constitution d'un domaine d'«études juives» en Roumanie est l'expression à la fois d'une nécessité d'intégration sociale et politique (et donc d'une éducation laïque) et d'une exigence d'assumer et continuer la culture juive. Elle est le résultat d'une rencontre entre une éducation laïque qui devient absolument nécessaire pour l'intégration et la tentative de maintenir un rapport vif avec la culture et la religion juive.

7 Les premières figures marquantes apparaissent au milieu du XIX ${ }^{e}$ siècle. Ainsi, Juliu Barasch (1815 -1863), immigrant de l'Autriche-Hongrie et un des pionniers de la médecine roumaine moderne, est le fondateur du premier périodique scientifique de Roumanie, Isis sau Natura (Isis ou la Nature), qui paraît entre 1856 et 1865, mais aussi le promoteur de l'affirmation de la culture et de l'identité juive. Parmi ses publications importantes, on peut mentionner la brochure parue en 1860 intitulée Emanciparea israelita în România (L'Émancipation israélite en Roumanie) ${ }^{7}$ et la publication bilingue (roumaine - française) Israelitul român (L'israélite roumain), en 1857. En sa mémoire est créée en 1886 la Société historique Iuliu Barasch, qui a fonctionné cinq ans et a publié les Annales de la société historique Iuliu Barasch, promoteur important des études d'histoire juive en Roumanie. Juliu Barasch est aussi le fondateur de la première école juive moderne de Roumanie en 1852.

8 Un autre pionnier des études historiques juives est Jacob Psantir ${ }^{8}$ (1820-1901). Il est l'auteur des premières histoires (ou chroniques historiques) des Juifs de Roumanie: Divre ha-Yamim la-Artzot Rumania, publiée à Jassi en 1871, et Korot ha Yehudim beRomania, publiée en 1873 à Lemberg (et traduite en roumain en 1877). Elles représentent le résultat d'un travail de terrain, de recherche et d'interprétation des inscriptions tombales de diverses régions du pays. Une chronique dont certaines informations intéressantes restent encore non explorées. Ses deux autres œuvres: sur la magie populaire (HaKesem) et sur la tolérance religieuse en Roumanie (HaSavlanut haDatit beRomania) pourraient aussi représenter une valeur documentaire significative pour les historiens contemporains.

9 La fin du XIX ${ }^{e}$ siècle et le début XXe siècle sont marqués par les figures des linguistes et folkloristes juifs roumains, pionniers de ces domaines d'études en Roumanie, tels Moses Gaster, Heinrich Tiktin, Alexandru Graur, Moses Schwartzfeld, ou A. Candea. Leur activité de recherche, d'enseignement et de publication se déroule dans les institutions publiques et les périodiques spécialisés roumains, mais aussi dans des revues juives 
spécialisées. Les publications juives en Roumanie sont très bien représentées, en grande partie grâce à la participation juive dans l'activité journalistique roumaine. Grâce aux journalistes, comme Moses Schwartzfeld (qui fonde la publication juive Egalitatea (l'Égalité) et Elias Schwarzfeld, sont fondées des publications juives importantes, comme la Allgemeine Zeitung des Judentum et Neue Frei Presse. La presse juive de la fin du dixneuvième siècle, reflétant les deux principaux mouvements culturels et politiques de l'époque - l'intégration promue par la Haskalah et par la Wissenschaft des Judentums ${ }^{9}$ est le cadre qui permet l'épanouissement des recherches dans le domaine des études juives. Des publications comme le Calendrier pour les Israélites et L'annuaire pour les Israélites (1879 - 1899) témoignent de la naissance d'une démarche scientifique dans les études juives. Moses Gaster y publie des études et articles sur l'histoire, le folklore, et la mystique juive, sur les karaïtes ou les sabbathiens.

Par l'ampleur, le haut niveau et la tenue de son activité scientifique, Moses Gaster représente une des plus grandes figures européennes du judaïsme de l'époque. Sa contribution au développement de la linguistique, des études folkloriques et de l'enseignement en Roumanie est décisive. Crestomatia română (La Chrestomathie roumaine), reproduction de plus de deux cents manuscrits roumains du dix-septième et du dix-huitième siècle, représente une des œuvres fondatrices de la philologie roumaine. Ses recherches comprennent l'histoire du judaïsme, la linguistique (il maitrisait plus de quatorze langues), la littérature biblique, intertestamentaire et apocryphe, la langue et la culture samaritaines et celles du Proche Orient ancien. Il publie des textes midrashiques, des targoumim, des textes de la Genizah du Caire ou des textes inédits comme le texte magique L'Épée de Moise ${ }^{10}$, il traduit le Siddur en roumain et écrit des manuels pour les écoles juives. Expulsé par le gouvernement Bratianu en 1885, il développa son activité scientifique à Oxford. Grand rabbin de la communauté sépharade d'Angleterre entre 1887 et 1919, il refuse de revenir en Roumanie après l'annulation du décret d'expulsion, mais continue à visiter la Roumanie et à s'intéresser à la langue, au folklore et à la littérature roumaine. En 1929 il devient membre de l'Académie roumaine. Le fonds Moses Gaster de la Bibliothèque de l'Académie roumaine, que le savant a donné à cette institution, comprend 750 textes et manuscrits, pour la plupart inédits. Une partie de ses articles publiés en Roumanie et en Angleterre ont été réédités à Londres entre 1925 et 1928. ${ }^{11}$ Beaucoup de ses recherches restent très actuelles ou sont redécouvertes à présent. Par exemple, sa vison du Zohar comme résultat d'une continuité et d'une multiplicité de traditions attire à présent l'attention des chercheurs importants, comme Moshe Idel. ${ }^{12}$

11 La langue yiddish devient, elle aussi dans cette période un objet d'étude et s'affirme comme langue littéraire. On assiste à une prise de conscience, suite au phénomène d'assimilation linguistique, du fait que la langue yiddish doit être conservée et cultivée. Des créateurs importants de langue yiddish, comme Moïse Ronetti Roman (Aharon Blumfeld), Iacob Groper, Itsik Manger, Jacob Sternberg, commencent leur activité littéraire dans la deuxième moitié du XIX $\mathrm{X}^{\mathrm{e}}$ siècle. Un théatre yiddish est ouvert à Bucarest par Jacob Sternberg. À Chisinau (en Bassarabie) paraît un journal yiddish, Cheser Zeit, et à Bucarest est fondé l'hebdomadaire littéraire yiddish Die Woch. En 1908 à Cernauti (en Bucovine) est organisé le premier congrès international pour la langue yiddish.

12 Dans la période 1918 - 1940 commencent à se constituer les premières sociétés et instituts d'études juives de Roumanie. Le rabbin M A Halevy, qui avait fait ses études à 
la Sorbonne, pionnier dans plusieurs domaines ${ }^{13}$, fonde en 1926 la Société d'Études juives de Roumanie, avec son périodique, Sinaï, ainsi que L'institut juif-roumain. À partir de 1928 le périodique devient un annuaire et continue à paraître jusqu'en 1933. M.A. Halevy manifeste un grand intérêt pour les archives historiques des Juifs de Roumanie et entreprend une des premières démarches de préservation, de classification et de recherche d'archives. Parmi les quelques institutions d'études juives, on mentionne l'institut du Temple Coral de Bucarest dans le cadre duquel commence à fonctionner en 1936 un cénacle d'études juives, présidé par I. Brucar.

Dans la Roumanie d'entre les deux guerres, les Juifs représentaient le groupe ethnique le plus scolarisé, très peu d'entre eux restant en dehors du système d'enseignement ${ }^{14}$. Dans certaines écoles en Moldavie le nombre d'élèves juifs dépassait celui des Roumains ${ }^{15}$. Ainsi, si on prend l'exemple de l'enseignement universitaire pour l'année 1928-1929, des données concernant la répartition par ethnie des étudiants inscrits dans les universités roumaines, aussi bien que des données statistiques (pour la période 1921-1933) offrent, à partir de l'appartenance ethnique, des informations concernant les domaines d'études prépondérants. Selon ces données, dans l'année universitaire 1928-1929 il y avait dans l'enseignement supérieur 30228 étudiants. Parmi eux, 24144 étaient Roumains, 4295 Juifs, 624 Hongrois, 433 Allemands, 253 Russes et 219 Bulgares :

Tableau 1 : La répartition des étudiants par ethnies entre 1928 et $1929^{16}$.

\begin{tabular}{|l|l|l|}
\hline & Nombre d'étudiants & Pourcentage du total \\
\hline Roumains & 24144 & 79,9 \\
\hline Juifs & 4295 & 14,2 \\
\hline Hongrois & 624 & 2,1 \\
\hline Allemands & 433 & 1,4 \\
\hline Russes & 253 & 0,8 \\
\hline Bulgares & 219 & 0,7 \\
\hline Autres & 260 & 0,9 \\
\hline Total & 30228 & 100,0 \\
\hline
\end{tabular}

Le pourcentage de la présence juive dans l'enseignement supérieur garde une valeur constante si on se rapporte à la deuxième recherche statistique mentionnée (tableau 2). En partant de ces données, on peut facilement remarquer la supériorité de la présence juive par rapport aux autres minorités nationales (14,2\% par rapport aux pourcentages inférieurs au $3 \%$ dans le cas des autres minorités nationales) et la visibilité accrue que cela confère à cette minorité. 
Tableau 2 : La répartition des étudiants dans les facultés, par ethnies entre 1921 et $1933^{17}$.

\begin{tabular}{|l|l|l|l|}
\hline & Roumains (\%) & Juifs (\%) & Autres nationalités (\%) \\
\hline Toutes les facultés & 77,1 & 16,4 & 6,5 \\
\hline Droit & 77,0 & 16,5 & 6,5 \\
\hline Lettres & 81,5 & 11,2 & 7,3 \\
\hline Sciences & 81,3 & 11,8 & 6,9 \\
\hline Médecine & 67,9 & 26,8 & 5,3 \\
\hline Théologie & 96,3 & - & 3,7 \\
\hline Pharmacie & 40,0 & 51,1 & 8,9 \\
\hline Médicine vétérinaire & 80,0 & - & 20,0 \\
\hline
\end{tabular}

Pour comprendre l'importance de cet élément, il faut considérer brièvement les transformations idéologiques dont l'environnement politique roumain souffre après la Première Guerre mondiale. On a affaire à une période où l'idée nationaliste devient dominante au niveau du discours politique, la majorité des militants nationalistes partageant l'aspiration d'un État roumain unitaire, homogène, qui comprenne tous les Roumains et uniquement les Roumains ${ }^{18}$. Cette aspiration contredit les réalités, multiculturelles et plurirégionales de la Roumanie unifiée : un pays pluriethnique où la communauté juive est la plus visible à la fois par le degré important d'urbanisation et par sa présence en grand nombre dans les institutions d'enseignement, particulièrement dans l'enseignement supérieur. Si on rajoute l'image - profondément enracinée- du Juif comme élément inassimilable ${ }^{19}$ ainsi que la définition de la reconquête des villes et la construction d'une élite exclusivement roumaine comme fondement de la mission nationale, on entrevoit le mécanisme de la transformation du Juif en un symbole de tout ce qui n'est pas Roumain et, implicitement, de tout ce qui constitue un obstacle pour l'accomplissement de l'idéal national.

16 Comme une conséquence de cette image, le Juif devient, à partir des années 1920, le sujet des discours nationalistes qui avaient comme but l'introduction du principe du numerus clausus dans le système d'enseignement roumain, principe qui représente un des éléments doctrinaux fondamentaux du Mouvement Légionnaire, ainsi que de la Ligue de la Défense nationale chrétienne dont le dirigeant principal était A.C. Cuza ${ }^{20}$. Les discussions autour de ces idées représentent une constante du discours nationaliste et il est le prétexte des manifestations antisémites dans les institutions universitaires de Roumanie entre 1920 - $1930^{21}$.

17 Le principe de numerus clausus devient loi le 29 août 1940 par une décision du Ministère de l'Éducation nationale, selon laquelle on n'admettait pas dans une classe plus de $6 \%$ d'élèves d'origine juive ${ }^{22}$. Mais les choses ne s'arrêtent pas là. Le 11 octobre 1940, le décret-loi numéro 3438 concernant la réglementation de la situation des Juifs dans le système d'enseignement prévoit l'exclusion des Juifs de toutes les formes et de tous les grades de scolarisation. L'article 3 de cette loi stipulait : «ceux qui sont nés des deux 
parents juifs, indifféremment de leur religion (...) ne sont pas admis comme élèves ou étudiants dans les écoles roumaines primaires, secondaires et supérieures, d'État ou privées et ne sont pas non plus admis dans les écoles des autres éléments ethniques chrétiens $»^{23}$. La loi permettait pourtant aux Juifs l'organisation, dans le cadre de l'enseignement privé, d'écoles primaires et secondaires propres, ayant un personnel didactique et administratif juif. L'origine de ces écoles date, comme déjà mentionné, de 1851. Elles ne pouvaient pas être fréquentées par des élèves autres que Juifs et les diplômes qu'elles avaient le droit de délivrer n'étaient pas reconnus par l'État et n'avaient pas de validité pour l'admission dans un domaine professionnel ${ }^{24}$.

Avec l'exclusion des élèves des écoles d'État et privées non juives, les professeurs en sont aussi exclus, comme tout le personnel administratif d'origine juive. Le même article du Décret-loi du 11 octobre 1940 prévoit : «Ceux qui sont nés de deux parents juifs ou ayant seulement le père juif, indifféremment de religion, ne peuvent pas travailler comme personnel enseignant ou administratif dans les écoles roumaines, ni dans les écoles des autres éléments ethniques chrétiens $»^{25}$. Pour toute cette période, l'enseignement juif entier, en incluant les salaires des cadres enseignants et du personnel administratif, est financé par les institutions communautaires juives et survit grâce aux taxes scolaires payées par les étudiants et aux ressources de financement propres.

La période difficile de 1940-1944 est dominée par la grande figure d'Alexandre Safran, qui devient grand rabbin en 1940, à l'âge de 30 ans. Docteur en philosophie de l'Institut de Hautes Études théologiques de Vienne et l'un des plus remarquables promoteurs d'une conscience culturelle juive, il sera expulsé en 1947 par le régime communiste. En tant que chef de communauté, il réussit pendant la guerre à sauver des vies, à faire annuler des décisions de déportation, à supprimer dans certaines régions l'étoile jaune, mais aussi à fonder de nouvelles écoles et de nouveaux lycées pour les élèves juifs qui n'étaient plus acceptés dans les institutions publiques. Il pose même les fondations d'une université juive qui ne survivra pas longtemps (L'École Polytechnique Bercovici). 26

Par ce que William Oldson a nommé un «antisémitisme providentiel $»^{27}$, plus de la moitié de la population juive roumaine a survécu à l'Holocauste. Si en 1939 la population juive de Roumanie était d'approximativement 800000 personnes, en 1945 il en restait 430000 personnes. Malgré le nationalisme sauvage d'un pays que Hannah Arendt considérait «le pays le plus antisémite de l'Europe d'avant la guerre $\aleph^{28} \mathrm{ou}$, paradoxalement, précisément grâce à ce nationalisme, le gouvernement roumain nationaliste-fasciste $d u$ maréchal Antonescu n'a pas cédé entièrement et inconditionnellement «ses " Juifs au Reich. Mais pour les survivants le début de la période communiste est loin d'être la fin des souffrances.

21 L'antisémitisme et la suspicion continuent, sous des formes différentes, pendant les premières années du régime communiste, un régime qui n'a jamais condamné le génocide et les déportations ${ }^{29}$ et qui a toujours eu des difficultés en ce qui concerne la définition et d'une population "potentiellement dangereuse ». L'antisémitisme revêt une forme nouvelle: la peur de "l'espionnage international sioniste ${ }^{30}$. Bien que des Juifs aient occupé des fonctions importantes dans les gouvernements communistes comme Ana Pauker, Iosef Chisinevshi et le redouté Leonte Rautu, le directeur du bureau de la propagande, une des figures les plus sinistres de la période communiste et le promoteur des violentes mesures antijuives, - la mentalité antisémite continue à être 
implicitement encouragée et alimentée par ces gouvernements. Au niveau de la population, les mythes et les préjugés continuent à être propagés (incroyablement, en 1965 on a même un cas d'accusation de meurtre rituel) ${ }^{31}$. La "question juive » est reformulée par les gouvernements communistes et cette permanente préoccupation pour une catégorie qui reste inassimilable et donc menaçante constitue une des raisons principales de la permission et l'encouragement de l'alya. Après l'instauration du régime nationaliste de Ceausescu, on arrête de parler d'un "problème juif » et à partir de 1970 le mot "juif» même devient tabou ${ }^{32}$. Encore une fois, le nationalisme est "providentiel»: Ceausescu trouve dans l'alya une solution au "problème juif» (de plus, une solution profitable).

La période communiste est marquée par la figure controversée de Moses David Rosen ${ }^{33}$, grand rabbin de Roumanie entre 1948 et 1994. Le rabbin Moses Rosen parvient à défendre les droits des Juifs roumains et à négocier avec les gouvernements communistes la liberté de faire la alya, mais aussi à créer un cadre nécessaire pour les activités culturelles de la communauté. À partir de 1959, des cours de Talmud Torah commencent à être donnés en demi-illégalité ${ }^{34}$. Moses Rosen réussit à leur donner un statut officiel. Il fonde en 1956 le magazine Cultul iudaic (Le culte juif), la seule publication de ce genre pendant le régime communiste, ainsi que le Musée de la Communauté Juive de Roumanie. Il réussit même à organiser des colloques et conférences d'études juives. Ainsi, malgré l'absence d'enseignements spécifiques ou d'institutions de recherche dans le domaine des études juives, il y a pendant l'époque communiste une activité culturelle communautaire qui permet à ceux qui n'ont pas choisi l'émigration de garder une continuité symbolique.

Après 1989, malgré la diminution considérable de la communauté (à présent, la communauté juive de Roumanie est estimée à moins de douze mille personnes), les études juives commencent à être représentées dans l'enseignement universitaire et dans la recherche des principales institutions d'enseignement supérieur de Roumanie. Actuellement fonctionnent à l'Université de Bucarest le Centre Goren Goldstein (programme de Master), fondé en 1998, et le Département d'études juives, fondé en 2002. À Cluj, la ville la plus importante de Transylvanie, est fondé en 1995 l'Institut d'études juives et d'histoire du peuple juif, Dr. Moshe Carmilly. Un Centre d'études juives fonctionne à l'Université de Iasi dans le cadre de la Faculté d'Histoire et un autre centre plus récent a été inauguré à Craiova. Le grand nombre d'étudiants qui choisissent ces cursus $^{35}$ - et qui sont majoritairement non-juifs - prouve l'intérêt grandissant pour un domaine jamais représenté auparavant dans les institutions universitaires de Roumanie.

Des instituts de recherche sur l'antisémitisme, comme l'Institut national pour la Recherche sur l'Antisémitisme Élie Wiesel de Bucarest, ou sur l'histoire juive, comme le Centre pour la Recherche sur l'Histoire des Juifs de Roumanie, organisent périodiquement des colloques internationaux sur des sujets d'histoire et de culture juive. Les activités d'édition et de publication sont également significatives: maisons d'édition importantes, comme Hasefer, périodiques, comme Caietele Institutului National pentru Studierea Holocaustului in Romania (Les Cahiers de l'Institut National pour l'Étude de l'Holocauste en Roumanie), édités par l'Institut National Élie Wiesel, Studia Hebraica, éditée par le Centre Goren Goldstein, ou Caietele Culturale ale Realitatii Evreiesti (Les Cahiers culturels de la Réalité juive), édités par la Fédération des Communautés juives de Roumanie. Les livres publiés (des centaines de titres) sont en 
partie des traductions - à partir des langues européennes ou de l'hébreu - de littérature, histoire, culture et religion juives et en partie des livres des auteurs juifs de Roumanie ou des auteurs juifs émigrés pendant la période communiste. Quatre musées d'histoire juive fonctionnent actuellement : le Musée d'Histoire des Juifs de Roumanie (à Bucarest), le Musée de l'Holocauste (à Bucarest), le Musée de la Communauté juive de Bacau et le Musée de la Communauté juive de Iasi. La Fédération des Communautés juives de Roumanie a une bibliothèque centrale à Bucarest et vingt autres bibliothèques locales. Les activités d'enseignement et de publication, les manifestations culturelles et communautaires démontrent que l'intérêt pour les études juives s'accroît en Roumanie malgré la diminution de la communauté locale et le « silence » qui a couvert le domaine dans la période communiste.

\section{BIBLIOGRAPHIE}

ARENDT, Hannah (1963), Eichmann in Jerusalem, Viking Press, New York.

BRATESCU, Gheorghe (2003), « Au centenaire de la naissance du grand rabbin Dr. Meyer Halévy. La contribution de M. A Halévy à l'historiographie médicale » dans Revue des Études juives, vol. 126/ janvier-juin 2003, pp. 247-251.

BRATULESCU, Monica (1993), “A Critical Approach to the Writtings of Jacob Psantir”, Shvut, 16, pp. 219-233.

GASTER, Moses (1925-1928), "Studies and Texts" in Folklore, Magic, Mediaeval Romance, Hebrew Apocrypha and Samaritan Archaeology, vol. 1-3, London, Maggs Bros.

HEINEN, Armin (2006), Legiunea Arhanghelului Mihail (La Légion de l'Archange Michael), Bucarest, Humanitas.

IANCU, Carol (1996), Les Juifs de Roumanie, 1919 - 1938. De l'émancipation à la marginalisation, Éditions Poche, 1996.

IANCU, Carol (2006), Alexandru Safran. Une vie de combat, un faisceau de lumière, Collection "Sens ", Université Paul Valéry - Montpellier III.

IDEL, Moshe (2007), “Moses Gaster on Jewish Mysticism and the Zohar" dans Tel Aviv University's Teuda, vol. XXI - XXII, ed. Ronit Meroz..

LIVEZEANU, Irina (1998), Cultura si nationalism in Romania Mare 1918 - 1930 (La culture et le nationalisme dans la Grande Roumanie 1918-1930), Bucarest, Humanitas.

OLDSON, William O. (1991), A Providential Anti-Semitism. Nationalism and Polity in Nineteenth Century Romania, Philadelphia, the American Philosophical Society.

STANCIU, Mariuca (2002), "The Press as a Path to Self-Emancipation. Stages in the Evolution of the Jewish Press in the 19th Century Romania" dans Studia Hebraica, no. 2, pp. 86-96.

VOLOVICI, Leon (1991), Nationalist Ideology and Antisemitism: The Case of Romanian Intellectuals in the 1930s. Oxford, Pergamon Press. 
VOLOVICI, Leon (1999), “National Communism and Jewish Politics: Rabbi Moses Rosen's Miracles and Dilemmas" dans Jewish Centers and Peripheries, ed. Ilan Troen, New Brunswick, pp. 85-98.

The History of the Jews in Romania, vol. I-IV, Tel Aviv, Goren Goldstein Diaspora Research Center, 2005.

The Final Report of the International Commission of the Holocaust in Romania Presented to the Romanian President Ion Iliescu (Le Rapport Final de la Commission Internationale de l'Holocauste en Roumanie présenté au Président roumain Ion Iliescu), Novembre 2004, Yad vaShem : http:// www.yadvashem.org/

\section{NOTES}

1. Signé à Paris, 9 décembre 1919.

2. Année de l'unification des principautés roumaines avec la Transylvanie, la Bessarabie et la Bucovine.

3. Apud Iancu, Carol (1996).

4. Une des raisons de tension inter - communautaire en Transylvanie. Cf. The Final Report of the International Commission of the Holocaust in Romania Presented to the Romanian President Ion Iliescu (Le Rapport Final de la Commission Internationale de l'Holocauste en Roumanie présenté au président roumain Ion Iliescu), Novembre 2004, texte disponible sur le site de Yad vaShem: www.yadvashem.org.

5. Ibid.

6. Ibid., p. 96.

7. Iancu, Carol, op. cit., pp. 71-72.

8. Sur Jacob Psantir, voir : Bratulescu, Monica (1993), pp. 219-233.

9. Voir : Stanciu, Mariuca (2002), pp. 86-96.

10. La Première édition : Londres, David Nutt, 1896.

11. Voir : Gaster, Moses (1925 - 1928).

12. Voir : Idel, Moshe (2007).

13. Voir : l'article de Bratescu, Gheorghe (2003), pp. 247-251.

14. Voir : Livezeanu, Irina (1998), p. 281.

15. Voir : Rotman, Liviu (1999), p. 95.

16. Apud Livezeanu, Irina (1998), p. 282.

17. Voir : Livezeanu, Irina, p. 282.

18. Idem, p. 353.

19. Conception largement disséminée, un leitmotiv du discours nationaliste spécifique pour la période d`entre les deux guerres mondiales. Voir, par exemple : A.C.Cuza, Nationalitatea in arta (La nationalité dans l'art), , Bucarest: Minerva, 1907, p. 69; Nichifor Crainic, Ortodoxie si etnocratie (Orthodoxie et etnocratie), Bucarest, Cugetarea, p.125; Nae Ionescu (le leader de la jeune génération, proche du mouvement nationaliste-fasciste roumain), la Préface au roman de Mihail Sebastian, De doua mii de ani... (Depuis deux mille ans), Bucarest, Humanitas, 2006, p. 8-9. Confer : Mihail Sebastian, Jurnal 1935-1944. Pour l'édition française : Journal, trad. Alain Paruit, Paris, Stock, 1998.

20. A.C. Cuza (1857 - 1947) est le créateur avec Nicolae Iorga du Parti nationaliste démocratique (1910), ayant un programme politique antijuif et, en 1922, de l'Union Nationale chrétienne, parti d'inspiration fasciste et paramilitaire. Il devient l'associé de Corneliu Zelea Codreanu entre 1923 et 1930 et en 1937 pour quelques mois ministre d'État dans le gouvernement catastrophique Cuza - Goga. Il est, par sa politique et son idéologie farouchement nationaliste et antisémite, une des figures principales de l'Holocauste en Roumanie. 
21. Le plus connu des épisodes de violence antisémite dans les universités a lieu au printemps de l'année 1923 et il a comme résultat l'arrêt de l'activité universitaire pour l'entière année. Pour plus de détails voir : Armin Heinen, Legiunea Arhanghelului Mihail (La Légion de l'Archange Michael), Bucarest, Humanitas, 2006.

22. La Décision. 153377 din 29 august 1940.

23. Le Rapport Final de la Commission internationale de l'Holocauste.

24. Idem.

25. Décret-Loi no. 3438 de 11 octobre 1940.

26. Voir : Iancu, Carol (2006).

27. Voir : O. Oldson, William (1991).

28. Voir: Arendt, Hannah (1963), p. 172.

29. Voir : The History of the Jews in Romania, (2005),Vol. 4, p. 116.

30. Ibid., pp. 156-166.

31. Ibid., p. 157.

32. Ibid., p. 166.

33. Moses David Rosen (1912 - 1994), grand rabbin des communautés juives de Roumanie entre 1948 et 2004, soutien du régime communiste, député dans la Grande Assemblée nationale et proche du président Ceausescu. Il convainc les autorités communistes de permettre la alyah d un très grand nombre de Juifs roumains et défend avec succès leurs droits. Cf. : Leon Volovici, “National Communism and Jewish Politics : Rabbi Moses Rosen's Miracles and Dilemmas" dans Jewish Centers and Peripheries, ed. Ilan Troen, New Brunswick, 1999, pp. 85-98.

34. Liviu Rotman, op.cit., p. 51.

35. Dans les centres de Bucuresti, Cluj, Iasi et Craiova, des dizaines d'étudiants suivent les programmes universitaires de licence, maîtrise et master. Même si le gouvernement finance des places spéciales pour les membres de la communauté juive, une petite minorité des étudiants appartient à la communauté.

\section{RÉSUMÉS}

The history of Jewish Studies in Romania is inseparable from a history of exclusions, persecutions, deportations and mass murder. However, systematic exclusions and marginalization, as well as the tardy and repeatedly restricted access to the public education system do not prevent Romanian Jewish community from bringing an important contribution to the progress of the local cultural and scientific life. The crystallization of a field of Jewish Studies is - here, as elsewhere in Europe, - the result of an encounter between a lay education, indispensable to the integration process, and the attempt to maintain a living contact with Jewish cultural and religious heritage. 
INDEX

מילות מפתח

יהודים ברומניה, המאה העשרים, ההוראה, בתי ספר ציבוריים, השתלבות, משה:

גסטר, אלכסנדר ספרן, משה דוד רוזן, רומניה

Keywords : twentieth century, nineteenth century, Barasch Juliu (1815 -1863), Psantir Jacob

(1820-1901), Gaster Moses (1865-1939), Safran Alexandre (1910-2006), Rosen Moses David

(1912-1994), Jews in Romania, teaching, public schools, integration, Romania

Index chronologique : vingtième siècle, dix-neuvième siècle

Index géographique : Roumanie

Mots-clés : Juifs de Roumanie, enseignement, écoles publiques, intégration, Barasch Juliu (1815

-1863), Psantir Jacob (1820-1901), Gaster Moses (1865-1939), Safran Alexandre (1910-2006), Rosen

Moses David (1912-1994) 